\title{
Reducing the risk of Dengue with Proper Diagnosis, Treatment and Education of People
}

\author{
Sakure $\mathbf{S}^{1}$, Bhosale $\mathbf{S}^{2}$, Shewale $\mathbf{S}^{3}$ \\ ${ }^{1}$ Sunita Sakure, Assistant Professor, Department Of Microbiology, S.B.B. alias Appasaheb Jedhe College, Shukrawar \\ peth, Pune, Maharashtra, India, ${ }^{2}$ Sarika Bhosale, Assistant Professor, Department Of Microbiology, S.B.B. alias \\ Appasaheb Jedhe College, Shukrawar peth, Pune, Maharashtra, India, ${ }^{3}$ Swapnil Shewale, Department of Zoology, \\ University of Pune, Ganeshkhind Road, Pune, Maharashtra, India.
}

Address for Correspondence: Mrs Sunita Satish Sakure, Email: sakuresunita@gmail.com

\begin{abstract}
Aedes aegypti is known to play a significant role in the transmission of various dreadful diseases such as dengue fever, chikungunya and yellow fever. Dengue fever (DF) is primarily caused by dengue fever virus (DENV). As per state health department report released in May, 2014 Maharashtra has reported 722 cases of dengue this year accounting for $25 \%$ of the dengue cases around the country. DENV serotypes are majorly transmitted by infected female mosquito that takes a blood meal from an infected person with DF. During the initial 2-10 day febrile period, DENV spreads within the body of the mosquito infecting the gut lining and later to salivary gland. Mosquito lay their eggs in artificial and natural stagnant water containers. When an infected female mosquito bites a person the virus enters the skin with the mosquito's saliva and infects leucocytes and reproduces inside these cells. The leucocytes respond by producing cytokines and interferons, causing high fever and severe pains. In severe infection, the virus invades organs like liver and bone marrow thereby lowering the blood pressure and internal bleeding leading to a risk of dengue hemorrhagic fever and dengue shock syndrome. Dengue NS-1 Antigen test is confirmatory for early and immediate diagnosis of dengue. The state of infection can be monitored by examination of platelet counts. As there is no antiviral drug discovered against dengue, so causing serious damage to people of all age groups. People should be educated and awareness should be carried out to overcome such a disease.
\end{abstract}

Keywords: Aedes Aegypti, Dengue, Platelet Count, Septic Shock Syndrome.

\section{Introduction}

Dengue fever is an insect borne viral disease caused by dengue virus (DENV) transmitted by female Aedes aegypti mosquito to human in a viral cycle that requires both human and these mosquitoes [1- 3]. Once a mosquito is infected, it remains infected for whole lifespan (25 to 30 days) [2,3]. Dengue viruses (DENV) belong to the family Flaviviridae, genus Flevivirus, distinguished into four serotypes (DENV-1, DENV-2, DENV-3, DENV-4) based on neutralization assay data [4]. Infection with any of the DENV serotype may be asymptomatic in majority of cases or may result in a wide spectrum of clinical symptoms, ranging from a mild flu like syndrome known as dengue fever (DF) to

Manuscript received: $1^{\text {st }}$ Dec 2014

Reviewed: $8^{\text {th }}$ Dec Aug 2014

Author Corrected: $19^{\text {th }}$ Dec 2014

Accepted for Publication: $28^{\text {th }}$ Dec 2014

International Journal of Medical Research and Review the most severe forms of the disease, which are characterized by coagulopathy, increased vascular fragility and permeability known as dengue hemorrhagic

fever (DHF) [4, 5]. DHF may progress to hypo-volemic shock known as dengue shock syndrome (DSS). Dengue fever is usually manifested as an incapacitating disease in people of all age groups. It is characterized by the rapid onset of fever in combination with severe headache, retro-orbital pain, myalgia, arthralgia, gastrointestinal discomfort and usually rash. Minor hemorrhagic manifestations may occur in the form of petechiae, epitaxis and gingival bleeding [5]. Leukopenia is a common finding, whereas thrombocytopenia is observed in DF especially in those with hemorrhagic signs. The World Health Organization (WHO) classifies DHF in four grades (I to IV) [6]. DHF

Available online at: www.ijmrr.in 106 | P a g e 
grades I and II represents relatively mild cases without shock, whereas grade III and IV cases are more severe and accompanied by shock. DHF is characterized by all the symptoms of DF in combination with hemorrhagic manifestations like positive tourniquet test or spontaneous bleeding, thrombocytopenia and evidence of increased vascular permeability characterized by increased hemo-concentration or fluid effusion in chest or abdominal cavities [7-10]. The life threatening DSS stage occurs at the time of or shortly after defervescence, which is characterized by a rapid, weak pulse $(\leq 20 \mathrm{mmHg}$ ) or hypotension with cold clammy skin in the early stage of shock (grade III). If patients do not received prompt and appropriate treatment, a stage of profound shock may set into undetectable pulse and blood pressure (grade IV) resulting in death within 1236 hours after the onset of shock $[11,12]$.

The treatment of dengue fever is symptomatic and supportive in nature $[3,13]$. The important part of the treatment is to eliminate body pain and control the fever by avoiding extra non necessary medications which results into an increased risk for hemorrhage and mortality. The patient's hydration status during the early febrile phase of illness can be monitored by performing haemo-dynamic assessments like baseline hematocrit testing and platelet counts, preventing complications such as prolonged shock and metabolic acidosis. Bed rest and mild analgesic-antipyretic therapy are often helpful in relieving lethargy, malaise and fever associated with the disease. Patients with dengue hemorrhagic fever or dengue shock syndrome may require intravenous volume replacement. Plasma volume expanders can be used in patients who are unresponsive to isotonic fluids $[14,15]$.

\section{Laboratory Diagnosis}

Laboratory diagnosis of Dengue can be done either by isolating virus or by detecting dengue specific antibodies [16]. For DENV detection, RNA of serum specimens are extracted and serotype specific reverse transcriptase polymerase chain reaction (RT-PCR) is carried within 5 days of symptom onset $[17,20]$. If the virus is not isolated / detected, a convalescent phase serum is required at least 6 days after the onset of symptoms to make a serological diagnosis by testing for IgM antibodies to dengue with an IgM antibody capture enzyme- linked immune-sorbent assay (MAC-ELISA) $[18,19,20]$. Non structural protein 1 (NS1) antigen detection kits are now commercially available. NS1 is a glycoprotein produced by all flaviviruses and is essential for viral replication and viability secreted into International Journal of Medical Research and Review the blood stream. Dengue NS-1 Antigen test or Dengue day 1 test is a rapid solid phase immunochromatographic test for the qualitative detection of Dengue NS1- Antigen and differential detection of IgM and $\mathrm{IgG}$ antibodies to dengue virus in human plasma or serum [21-25]. This test is for in vitro diagnostic use only and is intended as an aid in the earlier diagnosis of dengue infection and presumptive diagnosis between primary and secondary dengue infection.

\section{Principle (Antigen-antibody reaction)}

Dengue NS1 test in which colloidal gold complexes containing dengue 1-4 antigens prepared from dengue virus culture is captured by the bound anti-dengue IgM or IgG on respective test bands located in the test window causing a pale to dark red band to form at the $\mathrm{IgG}$ or IgM region of the test device window. The intensity of the test bands in the respective device will vary depending upon the amount of antigen/ antibody present in the sample. The appearance of any pink/red colour in a specific test region should be considered as reactive for that particular antigen and/or antibody type (IgG or $\operatorname{IgM}$ ). A red procedural control line should always develop in the test device window to indicate that the test has been performed properly.

\section{Specimen collection and Preparation [25,26]}

Serum / plasma samples may be used with this test. The use of hemolytic, lipemic, icteric or bacterially contaminated samples should be avoided as it may lead to erroneous results.

\section{Test procedure}

\section{Dengue NS1 Antigen Device:}

Add 3 drops $(100 \mu \mathrm{l})$ of sample (serum/ plasma ) using Dengue Antigen Test sample dropper to the sample well of antigen device and incubate it for 20 minutes and then analyse the result. Positive results may appear as early as 2-10 minutes. However, negative results must be confirmed after 20 minutes only.

\section{Dengue IgM \& IgG Antibodies Device:}

Fill the Dengue Antibody lower circular part of the sample dropper with the sample (serum/plasma) up to the mark provided on the dropper. Then add the sample to the sample well "S" of antibody device. This will add $10 \mu \mathrm{l}$ of sample (serum/plasma) to the device. Dispose of the dropper considering it to be bio-hazardous. Incubate for 20 minutes and confirm the results.

\section{Interpretation of the test}

Available online at: www.ijmrr.in 107 | P a g e 


\section{A Dengue NS1 Ag Device}

Reactive: Appearance of pink colored line, one each in test region " $\mathrm{T}$ " and control region " $\mathrm{C}$ " indicates that the sample is REACTIVE for Dengue NS1 Ag.

Non-Reactive: Appearance of one distinct pink line in the control region " $\mathrm{C}$ " only, indicates that the sample is "NON-REACTIVE" for Dengue NS1 Ag.

Invalid: When neither control line nor the test line appears on the membrane the test is treated as invalid.

B Dengue IgM \& IgG Antibodies Device

IgM \& IgG Reactive: Appearance of red colored line in the control region ' $\mathrm{C}$ ' and Test region; IgM region ' $M$ ' and IgG region ' $G$ ' indicates that the sample is reactive for both IgM \& IgG antibodies. This is indicative of a secondary dengue infection.

IgM Reactive: Appearance of red colored line in the control region ' $\mathrm{C}$ ' and Test region ; IgM region ' $\mathrm{M}$ ' indicates that the sample is reactive for IgM antibodies. This is indicative of a primary dengue infection.

IgG Reactive: Appearance of red colored line in the control region ' $c$ ' and test region; IgG region ' $G$ ' indicates that the sample is reactive for IgG antibodies. This is indicative of a secondary dengue infection.

Non Reactive : Appearance of one distinct red color line in the control region ' $c$ ' only with no line in the IgM region $M$ and $I g$ G region ' $G$ ' indicates that the sample is non reactive for dengue antibodies.

Invalid: When neither control line nor the IgM / IgG line appears the test should be treated as invalid. It may be because of improper storage at temperature other than recommended temperature, wrong procedure or long atmospheric exposure of the test device after opening the kit.

\section{Treatment}

Generally dengue complications are often termed as dengue shock syndrome associated with high mortality rate, which can lead to vascular permeability, dehydration, myocardial dysfunction contributing to development of shock or even multi-organ failure [27]. The most dangerous thing about dengue is that there are no specific antiviral drugs discovered till today and because of this only the symptoms that can be treated.
The treatment involves mainly the part of maintaining the fluid balance to prevent dehydration which can be done with adequate rest and fluid intake. Large amounts of fluids (water, soup, milk, juice) along with patient's normal diet is recommended [27,28,29]. Acetaminophen (Paracetamol) and codeine can be given for severe headache and for joint and muscle pain. Taking drugs like aspirin, other salicylates, and non-steroidal antiinflammatory drugs (NSAIDs) are dangerous since the infecting organism in dengue affects the platelets which are responsible for the clotting of blood. Spontaneous decrease in platelet count increases a person's tendency to bleed excessively resulting in DSS. Prevention is the best option as there are no specific treatment options. Dengue can be prevented by avoiding mosquito bites using mosquito repellents available in commercial medicals and druggists [30,31].

\section{Discussion}

As Aedes aegypti mosquitoes are known to bite humans during the day and their most common breeding grounds are manmade containers, it is advisable to not have stagnant water around. Turn over the buckets and pails which are not in use. Adoption of good daily habits such as clearing blockages from the roof gutter, clearing leaves and stagnant water from drains, removing water from potted plants daily, avoiding the use of pot plates and changing the water in vases everyday will also help to eliminate the chances of mosquito breeding. Windows and door screens do not have any holes make sure it by blocking those areas properly to eliminate mosquitoes. If someone had dengue or dengue like symptoms, try to not let the mosquitoes bite them or others in the house. Empty and clean the cooler tray regularly, even when not in use. Natural methods to keep mosquitoes away are to plant Ocimum (tulsi) near windows which have properties that do not allow mosquitoes to breed. Light camphor as a repellent in a room for fifteen to twenty minutes to have a mosquito free environment. Do not throw Styrofoam cups after drinking tea or water into the dustbins. With a little bit of water accumulation, dengue mosquitoes can breed easily. Precautions include wearing long-sleeved clothes, using mosquito coils and electric vapour mats, and using insect repellent over the exposed parts of the body during the day as well as night.

\section{Antivirals research work under investigation}

The overall annual burden of dengue as reported by World Health Organization (WHO) is an estimated 50100 million dengue infections and 5,00,000

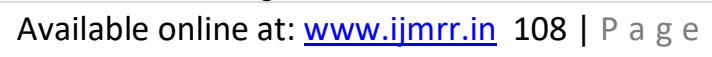


hospitalizations for severe form of the disease across the globe $[11,12]$. This burden is projected to continue to increase day by day. Several live attenuated dengue candidate vaccines are proceeding through various clinical evaluations. The need to induce a balanced immune response against all four DENV serotypes with a single vaccine has been a challenge for dengue vaccine developers. A live attenuated DENV chimeric vaccine produced by Sahofi Pasteur has recently entered phase 111 evaluation in numerous dengue endemic regions of the world showing $88.5 \%$ efficacy after three doses against severe disease dengue haemorrhagic fever which leads to hospitalization for over half a million people (mostly children) anually. Once administered, the vaccine also provided $67 \%$ protection against dengue-associated hospitalization. Researchers also found that the vaccine gave low protection (35\%) against DENV 2 strain, but more than $75 \%$ protection against DENV 3 and 4, and 50\% against DENV 1.

Each of the four distinct serotypes is capable of causing the full spectrum of dengue illness but epidemiological studies have determined that the risk for more severe dengue illness is higher following a second, heterotypic DENV infection than for a primary DENV infection. Although severe dengue illness can occur with a third or fourth DENV infection, this risk appears to be very low. For these reasons, there is urgency of a successful DENV vaccine which must ideally protect against all four DENV serotypes with a limited number of doses given over a period of weeks to a few months. This vaccine should be available to high risk group at an affordable cost. Till date several novel dengue vaccines have been developed including DNA vaccines, viral vector and protein subunit vaccines [32-35].

\section{Conclusion}

Improper treatment of a dengue patient can result into complications associated with dengue which usually appears between the third and fifth day of fever. Although fever subsides patients should be monitored closely for other life threatening signs for another two days. So if patients have symptoms like bleeding from nose or gums, frequent vomiting, vomiting with blood, black stool, abdominal pain, difficulty in breathing should immediately consult a doctor. Even though fever subsides patients should continue to monitor platelet count till the drop in count stops. Platelet count below 20,000 has higher chances of developing bleeding complications as seen in dengue hemmorrhagic fever. There is also a typical viral fever in which even though patients have all these symptoms, they are testing negative for dengue when specific antibody tests like Dengue IgG and Dengue IgM are conducted indicating that the typical viral fever is on the rise. In this case try to keep body temperature to normal and monitor continuous platelet counts at specific time intervals till the platelet counts rises to normal ranges up to $150-450$ x $10^{3}$ cells $/ \mu \mathrm{L}$ in peripheral blood examination.

\section{Future Aspects}

With the sustained dengue prevention and control measures in high risk places, the number of dengue cases will probably remain lower ultimately causing low deaths. The launching of dengue prevention campaign is a serious need to destroy the possible breeding sites of the dengue vector. There is also need of education of people regarding transmission, symptoms and treatment of the disease. This will definitely reduce the cases of life threatening Dengue in future.

\section{Conflict of interests}

The authors have declared no conflict of interest.

\section{References}

1. Scott C. Weaver, Alan D. T. Barrett. Transmission cycles, host range, evolution and emergence of arboviral disease. Nature Reviews Microbiology Oct 2004,(2):789-801. doi:10.1038/nrmicro1006.

2. Anna Drexler' Andrew Nuss' Eric Hauck' Elizabeth Glennon' Kong Cheung, Mark Brown' Shirley Luckhart. Human IGF1 extends lifespan and enhances resistance to Plasmodium falciparum infection in the malaria vector Anopheles stephensi. J Exp Biol. Jan 15,2013;216(2): 208-217. doi: 10.1242/jeb.078873.

3. Charles Patrick Davis, Dengue Fever, Medically Reviewed by a Doctor on 7/25/2014. eMedicinehealth. www.emedicinehealth.com/infections/center.htm.

4. Rosmari Rodriguez-Roche and Ernest A. Gould. Understanding the Dengue Viruses and Progress towards Their Control.BioMed Research International, Volume 2013 (2013); 1-20 Article ID 690835, http://dx.doi.org/10.1155/2013/690835

5. Harris E, Videa, E, Pérez L, Sandoval E, Téllez Y, Pérez ML, Cuadra R, Rocha J, Idiaquez W, Alonso RE, Delgado MA, Campo LA,Acevedo F, Gonzalez A, Amador JJ, Balmaseda A. . Clinical, epidemiologic, and virologic features of dengue in the 1998 epidemic in Nicaragua. Am. J. Trop. Med, Hyg, Jul-Aug 2000 63(1,2):5-11 
6. Byron E. E. Martina, Penelope Koraka and Albert D.M.E.Osterhas. Dengue Virus Pathogenesis: an Integrated View. Clincal Microbiology Review Oct 2009; 22(4): 564-581.

7. Linda S. Lloyd. Best Practices for Dengue Prevention and Control in the America. Environmental Health ProjectFebruary 2003.

www.ehproject.org/PDF/Strategic_papers/SR7 .

8. Global strategic framework for integrated vector management WHO, Geneva, Switzerland, world health organization 2004. World Health Organization 2004.

whqlibdoc.who.int/hq/2004/WHO_CDS_CPE_PVC_20 $04 \_10 . p d f$

9. Fifty-eight World Health Assembly Geneva. Resolutions and Decisions. 16-25 May 2005. apps.who.int/gb/ebwha/pdf_files/WHA58.../A58_2005_ REC1-en.pdf.

10. TDR/WHO. Dengue hemorrhagic fever: early recognition, diagnosis and hospital management. An audiovisual guide for health-care workers responding to outbreaks WHO, Geneva, Switzerland, 5 oct 2006. www.who.int/tdr/publications/documents/swg_dengue 2.pdf

11. TDR/WHO. Scientific working group report on dengue Meeting report. 1-5 October 2006, Geneva, Switzerland. Publication date: 2006/10/05 WHO reference number: TDR/SWG/08. www.who.int/tdr/publications/tdr-research.../swgreport-dengue/en/.

12. Dengue: Guidelines for Diagnosis, Treatment, Prevention and Control TDR/WHO,Geneva, Switzerland.1November 2009. WHO reference number: WHO/HTM/NTD/DEN/2009.

www.who.int/tdr/publications/disease/dengue/en/.

13. Bandyopadhyay S, Lum LC, Kroeger A. Classifying dengue: a review of the difficulties in using the WHO case classification for dengue hemorrhagic fever. Trop. Med. Int. Health 2006 (11):1238-1255.

www.ncbi.nlm.nih.gov/pubmed/16903887 - Cited by 162 - Related articles.

14. Dengue Guidelines for Diagnosis, Treatment, Prevention and Control. France: A joint publication of the World Health Organization (WHO) and the Special Programme for Research and Training in Tropical Diseases (TDR); 2009.

http://www.who.int/tdr/publications/ documents/ denguediagnosis.pdf.

15. Nguyen Thanh Hung, Nguyentrong Lan, HuanYaolei et al., Volume replacement in infants with Dengue haemorrhagic fever/Dengue shock syndrome
2006, Am. J. Trop. Med. Hyg., 2006 74(4):684-691. www.cdc.gov.tw/english/info.aspx?treeid=3847719104 be0678hungdhf@hcm.fpt.vn.

16. P. Philip Samuel \& B.K. Tyagi. Diagnostic methods for detection \& isolation of dengue viruses from vector mosquitoes. Indian J Med Res, May 2006;123(5):615628. icmr.nic.in/ijmr/2006/may/0508.pdf. Cited by 49 Related articles.

17. Li-Jung Chien, Tsai-Ling Liao, Pei-Yun Shu, JyhHsiung Huang et al., Development of Real-Time Reverse Transcriptase PCR Assays To Detect and Serotype Dengue Viruses. Journal of clinical microbiology, April 2006; 44(4):1295-1304. doi: 10.1128/JCM.44.4.1295-1304.2006

18. Guzman, M. G. \& Kouri, G. Dengue diagnosis, advances and challenges. International Journal of infectious diseases. March 2004, 8(2):65-136. DOI: http://dx.doi.org/10.1016/j.ijid.2003.03.003.

19. Gubler, DJ. Serologic diagnosis of dengue/dengue haemorrhagic fever. Nature Reviews Microbiology. 1996, (20) 20-23. doi:10.1038/nrmicro2460 .www.nature.com > Journal home > Archive > Review

20. Rosen L, Drouet MT, Deubel V. Detection of dengue virus RNA by reverse transcription-polymerase chain reaction in the liver and lymphoid organs but not in the brain in fatal human infection. Am. J. Trop. Med. Hyg Nov 1999,61(5):720-724.

www.ncbi.nlm.nih.gov/pubmed/10586901 by L Rosen 1999

21. Balmaseda A, Maria G, Guzman SH et al. Diagnosis of dengue virus infection by detection of specific immunoglobulin $\mathrm{M}(\operatorname{IgM})$ and $\operatorname{IgA}$ antibodies in serum and saliva. Clin. Diagn. Lab. Immunol 2003;(10):317322 .

22. Pei Yun Shu, Li-Kuang Chen, Shu-Fen Chang, YiYun Yueh, Ling Chow, Li-Jung Chien, Chuan Chin, Ting-Hsiang Lin, Jyh-Hsiung Huang. Comparison of capture immunoglobulin M ( IgM ) and IgG enzymelinked immunosorbent assay (ELISA) and nonstructural protein NS1 serotype-specific IgG ELISA for differentiation of primary and secondary dengue virus infections. Clin Vaccine Immunol July 2003,10(4):622630. doi: 10.1128/CDLI.10.4.622-630. 2003

23. Pei Yun Shu, Li-Kuang Chen, Shu-Fen Chang, YiYun Yueh, Ling Chow, Li-Jung Chien, Chuan Chin, Ting-Hsiang Lin, and Jyh-Hsiung Huang. Potential application of nonstructural protein NS1 serotype-specific immunoglobulin $G$. enzyme-linked immunosorbent assay in the seroepidemiologic study of dengue virus infection: correlation of results with those of the plaque reduction neutralization test. Clinical 
Microbiology May2002;40(5):1840-1844. doi: 10.1128/JCM.40.5.1840-1844.2002

24. Hua Xu, Biao Di, Yu-xian Pan, Li-wen Qiu, Ya-di Wang,Wei Hao, Li-juan He, Kwok-yung Yuen and Xiao-yan Che. Serotype 1-specific monoclonal antibody-based antigen capture immunoassay for detection of circulating nonstructural protein NS1: implications for early diagnosis and serotyping of dengue virus infections. J Clin Microbiol. Aug 2006; 44(8):2872-2878. doi: 10.1128/JCM.0077706.

25. Young, P. R., Hilditch, P. A., Bletchly, C. \& Halloran, W. An antigen capture enzyme-linked immunosorbent assay reveals high levels of the dengue virus protein NS1 in the sera of infected patients, J. Clin. Microbiol. March 2000,38(3):1053-1057.

26. Guzman MG, Kourig. Advances in dengue diagnosis, Clinical \& Diagnostic Laboratory Immunology Nov 1996, 3(6):621-627.

27. Senaka Rajapakse. Dengue shock. J Emerg Trauma Shock, Jan-Mar 2011; 4(1):120-127. doi: 10.4103/0974-2700.76835.

28. Halstead SB, Dengue. Lancet., Nov 10, 2007;370(9599):1644-52.

29. Halstead SB. Is there an inapparent dengue explosion? Lancet 1999 Mar 27;353(9158):1100-1101.

30. Centers for Disease control and prevention. National Center for Emerging and Zoonotic Infectious Diseases;2014. (Division of Vector-Borne Diseases (DVBD). www.cdc.gov/ncezid/dvbd/.

31. Robert Preidt. Avoid Mosquito Bites to Prevent Dengue Fever in Florida: Expert July 21, 2010. Loyola University news release, www.medicinenet.com infectious disease and news.health.com/2010.23 july 2010 .

32. Guirakhoo F, Kitchener S, Morrison D, Forrat R, McCarthy K, Nichols R, Yoksan S, Duan X, Ermak TH, Kanesa-Thasan N, Bedford P, Lang J, Quentin-Millet MJ, Monath TP. Live attenuated chimeric yellow fever dengue type 2 ChimeriVax-DEN2 vaccine: Phase I clinical trial for safety and immunogenicity: effect of yellow fever pre-immunity in induction of cross neutralizing antibody responses to all 4 dengue serotypes. Hum Vaccin. Mar-Apr 2006; 2(2):60-67. Epub 2006 Mar 15

33. Anna P. Durbin, Stephen S. Whitehead, Julie McArthur, John R. Perreault,Joseph E. Blaney Jr., Bhavin Thumar, Brian R. Murphy and Ruth A. Karron. rDEN4 $\Delta 30$, a Live Attenuated Dengue Virus Type 4 Vaccine Candidate, Is Safe, Immunogenic, and Highly Infectious in Healthy Adult Volunteers. The Journal of Infectious Diseases.2005;191(5):710-718.

34. Edelman R, Wasserman SS, Bodison SA, Putnak RJ, Eckels KH, Tang D, Kanesa-Thasan N, Vaughn DW, Innis BL, Sun W. Phase I trial of 16 formulations of a tetravalent live-attenuated dengue vaccine. Am J Trop Med Hyg. Mar 2004;70(3):48-60.

35. Peter F. Wright, Anna P. Durbin, Stephen S. Whitehead, Mine R. Ikizler, Susan Henderson, Joseph E. Blaney, Bhavin Thumar, Sharon Ankrah, Michael T. Rock, Brett A. McKinney, Brian R. Murphy, Alexander C. Schmidt. Phase 1 trial of the dengue virus type 4 vaccine candidate rDEN4 $\triangle 30-4995$ in healthy adult volunteers. Am. J. Trop. Med. Hyg: 2009; (81), 834841. doi:10.4269/ajtmh.2009.09-0131.

\section{How to cite this article?}

Sakure S, Bhosale S, Shewale S. Reducing the risk of Dengue with Proper Diagnosis, Treatment and Education of People. Int J Med Res Rev 2015;3(1):106-111. doi: 10.17511/ijmrr.2015.i1.17 